\title{
Retroperitoneal Sarcoma by AJCC v8 Stage
}

National Cancer Institute

\section{Source}

National Cancer Institute. Retroperitoneal Sarcoma by AJCC v8 Stage. NCI Thesaurus.

Code C136811.

A term that refers to the staging of retroperitoneal soft tissue sarcoma, following the rules of the TNM AJCC v8 classification system. This staging system applies to common sarcomas in the retroperitoneum. (from AJCC 8th Ed.) 